\title{
Failure of Ceftriaxone in an Intravenous Drug User with Invasive Infection Due to Ralstonia pickettii
}

\author{
C. Zellweger, T. Bodmer, M. G. Täuber, K. Mühlemann
}

\begin{abstract}
We report a case of septic arthritis due to Ralstonia pickettii in an intravenous drug user with unfavorable clinical course under antibiotic therapy with ceftriaxone despite in vitro susceptibility to the drug. The treatment failure may have been due to a discrepancy between in vitro and in vivo susceptibility of $R$. pickettii, or to resistance development mediated by a recently described inducible ß-lactamase.
\end{abstract}

Infection 2004; 32: 246-248

DOI 10.1007/s15010-004-3033-0

\section{Introduction}

Ralstonia pickettii, formerly known as Pseudomonas (Burkholderia) pickettii, is a nonfermenting gram-negative rod. On the basis of DNA-DNA and DNA-rRNA homology studies, the germ belongs to the Pseudomonas homology group II. $R$. pickettii is a ubiquitous environmental organism, which can be found in water, soil, and plants including fruits and vegetables. Nosocomial outbreaks due to contamination of intravenous solutions, "sterile" water, saline, chlorhexidine solutions, respiratory therapy solutions, and intravenous catheters with $R$. pickettii have been described [1]. This microorganism has also been associated with asymptomatic colonization of the respiratory tract as, for example, in cystic fibrosis patients [1]. Due to biochemical similarities, $R$. pickettii may be confounded with Burkholderia cepacia, which can cause exacerbations of pulmonary disease in patients with cystic fibrosis [2]. In humans, $R$. pickettii is an infrequent cause of invasive infections such as bacteremia [3, 4], meningitis [5], endocarditis [6] and osteomyelitis [7]. Here, we describe a patient with intravenous drug use, who developed severe invasive infection due to $R$. pickettii. Of note, we found a discrepancy between in vitro susceptibility to ceftriaxone and clinical response to therapy with this antibiotic. The reason for this phenomenon is unclear and may be due either to uncertainties about breakpoints, or to induction of resistance during antibiotic therapy.

\section{Case Report}

In October 2000, a 24-year-old male intravenous drug user was admitted to hospital for suspected intoxication. A few hours after admission he developed signs of severe septicemia and a stiff neck. White blood cell count $(11.9 \times 109 / 1)$ and C-reactive protein $(291$ $\mathrm{mg} / \mathrm{l}$ ) were elevated. A CT scan of the head was normal. Cerebrospinal fluid examination revealed pleocytosis of $106 \times 10^{6 / 1}$ with $86 \%$ polymorphonuclear cells, protein $0.74 \mathrm{~g} / 1$, glucose $4.91 \mathrm{mmol} / \mathrm{l}$; no microorganisms were seen on Gram staining and cultures remained sterile. Empirical antibiotic therapy with ceftriaxone, vancomycin, and amoxicillin for meningitis was initiated. Blood cultures grew Group A streptococcus. A transesophageal echocardiogram revealed a thickened anterior mitral valve with prolapse and a partially flail leaflet with moderate valve insufficiency, consistent with endocarditis. On the 2nd day of hospitalization, redness and swelling was noted in the area of the right elbow and the left knee. Both joints were surgically drained.

Aspirates of both joints yielded a gram-negative rod, which was identified as $R$. pickettii with the commercial gallery API $20 \mathrm{NE}$ (bioMérieux Vitek, Hazelwood, MO). The identification was confirmed by cellular fatty acid analysis using the Microbial Identification System (Microbial ID, Inc., Newark, DE). The antimicrobial susceptibility testing was performed according to the National Committee for Clinical Laboratory Standards for disk susceptibility testing. Susceptibility testing was done on Mueller-Hinton agar. The plates were incubated aerobically at $35^{\circ} \mathrm{C}$ without $\mathrm{CO}_{2}$ for $24 \mathrm{~h}$. By disk diffusion the microorganism appeared sensitive to piperacillin, piperacillin/tazobactam, higher generation cephalosporins (including cefuroxime, ceftriaxone, ceftazidime, cefepime), imipenem, trimethoprim-sulfamethoxazole, and ciprofloxacin. Intermediate sensitivity was found for aztreonam and netilmicin. In vitro resistance was noted for ampicillin/clavulanate, ticarcillin/clavulanate, meropenem, gentamicin, tobramycin, and amikacin. Minimal inhibitory concentrations (MIC)

\footnotetext{
C. Zellweger (corresponding author)
}

Division of Infectious Diseases, University Hospital, Inselspital PKT2 B, CH-3010 Bern, Switzerland; Phone: (+41/31) 632-0151, Fax: -3176. e-mail: claudine.zellweger@insel.ch.

T. Bodmer, M. G. Täuber, K. Mühlemann

Institute for Infectious Diseases, University Hospital Bern, Bern, Switzerland

Received: March 6, $2003 \cdot$ Revision accepted: March 30, 2004 
determined by E-test (AB Biodisk, Solna, Sweden) were 0.750 $\mathrm{mg} / \mathrm{l}$ for ceftriaxone and $0.047 \mathrm{mg} / \mathrm{l}$ for ciprofloxacin.

In accordance with culture results and antibiotic resistance testing, the treatment regimen was changed to ceftriaxone $2 \mathrm{~g} \mathrm{qd}$ for the endocarditis caused by Group A streptococcus and the septic polyarthritis due to $R$. pickettii. However, the clinical response to this therapy was unsatisfactory. Levels of C-reactive protein remained elevated at $114 \mathrm{mg} / \mathrm{l}$ and, after 2 weeks, purulent arthritis of the knee recurred, requiring repeated surgical drainage. $\mathrm{Cul}$ tures of joint aspirate remained sterile. The treatment regimen was changed from ceftriaxone to oral ciprofloxacin $500 \mathrm{mg}$ bid and penicillin G 24 Mio E qd. Under this regimen, the clinical and laboratory findings improved. Because of progressive heart failure, the mitral valve was replaced. A eubacterial polymerase chain reaction performed on the valve revealed DNA of Group A streptococcus; $R$. pickettii DNA was not detected.

The further clinical course was complicated by pericardial tamponade and partial thrombosis of the portal vein. The patient was discharged to further rehabilitation 8 weeks after admission. Two weeks later, however, he had to be transferred back to the hospital because of sternal wound infection with two different strains of methicillin-resistant Staphylococcus aureus. At that time, and at follow-up examination $1 \frac{1 / 2}{2}$ months later, arthritis of the right elbow and the left knee had healed without sequelae.

\section{Discussion}

Polymicrobial invasive infections are not uncommon in intravenous drug users. This patient had a dual infection with Group A streptococcus and R. pickettii. Group A streptoccocus was isolated from blood cultures and indirectly (eubacterial polymerase chain reaction) from the mitral valve, while $R$. pickettii was isolated from two different joint aspirates (elbow and knee). These samples were not collected in blood culture bottles, but inoculated on solid media. Also, the isolation of the pathogen from two different sites makes a contamination unlikely.

The involvement of two separate joints indicates that the patient experienced at least transient bacteremia with $R$. pickettii. Whether this pathogen also played a role in the endocarditis is not certain, but appears unlikely, based on the eubacterial polymerase chain reaction from the cardiac vegetation, which only documented Group A streptococcal genome. Along the same line, a contribution of the Group A streptococcus to the septic arthritis, while not ruled out, could not be documented microbiologically.

Based on published reports, $R$. pickettii rarely causes severe invasive infection. Several nosocomial outbreaks have been described in pediatric or oncology wards [8-10] and intensive care units [11] due to contamination of fluids. Most invasive infections seem to occur in patients with underlying diseases such as cancer, chronic renal failure, or they are related to foreign bodies (intravenous catheters). Here, we describe for the first time an invasive infection in an intravenous drug user. The source of infection was most likely the tap water, which the patient used to rinse his hypodermic needles.

The septic arthritis due to $R$. pickettii involving two separate joints showed an unfavorable clinical course under therapy with ceftriaxone, despite in vitro susceptibility of the organism initially isolated. Given the well-documented efficacy of ceftriaxone in the therapy of septic arthritis and bone infections, it is unlikely that this failure was caused by insufficient drug penetration into the infected joints. Rather it raises the question whether the in vitro testing for this organism, for which there is a lack of defined breakpoints, is predictive of the in vivo response.

It has been suggested that interpretative standards for Pseudomonas aeruginosa may be used for R. pickettii [1]. Therefore, a MIC of $0.750 \mathrm{mg} / 1$ for ceftriaxone would indicate susceptibility to this drug. The unfavorable clinical response in this case is reminiscent of the well-described disparity between in vitro MIC, and in vivo efficacy for $\beta$-lactam antibiotics with $P$. aeruginosa [12].

It is also conceivable that the organism developed resistance to $\beta$-lactam antibiotics during therapy with ceftriaxone. Although the resistance mechanisms in $R$. pickettii are not known in detail, intrinsic, acquired, and genetic resistance patterns similar to those of other gram-negative bacteria must be considered. Recently, Nordmann et al. [13] described a naturally occurring chromosomal and inducible Ambler class D B-lactamase, oxacillinase 22 (OXA-22), in a clinical isolate of $R$. pickettii. OXA-22-like genes were further identified in five other clinical isolates. In susceptibility tests where MICs of selected $\beta$-lactams were determined $R$. pickettii isolates were resistant, or had decreased susceptibility, against amino- and ureidopenicillins, restrictedspectrum cephalosporins, ceftazidime, and aztreonam, while susceptibility to ceftriaxone was not studied. Though not tested, it is reasonable to assume that ceftriaxone is also, at least partially, hydrolysed by OXA-22. In addition, the expression of OXA-22 is inducible in $R$. pickettii [13]. Unfortunately, development of resistance during therapy could not be tested, since the organism could not be recovered in the second joint aspirates.

In conclusion, $R$. pickettii, mainly known as an opportunistic pathogen of nosocomial infection, can cause severe invasive infection. Here, we describe the first case of septic polyarthritis due to $R$. pickettii in an intravenous drug user with a polymicrobial infection. The choice of the appropriate antibiotic regimen for infections due to $R$. pickettii, especially in the case of polymicrobial infections, may be difficult due to the lack of interpretative breakpoints for in vitro susceptibility tests, and scarce knowledge about the antibiotic resistance mechanisms used by this pathogen. Inducible $\beta$-lactamase activity in $R$. pickettii (mediated by oxacillinase OXA-22 or OXA-22-like $\beta$-lactamase) has to be considered. Fluoroquinolones may provide effective therapy when in vitro testing indicates susceptibility to this class of antibiotics.

\section{References}

1. Gilligan PH, Whittier S: Burkholderia, Stenotrophomonas, Ralstonia, Brevundimonas, Comamonas, and Acidovorax. In: Murray 
PR, Baron EJ, Pfaller MA, Tenover FC, Yolken RH (eds): Manual of clinical microbiology, (7th edn). ASM Press, Washington DC 1999, 526-538.

2. Gilligan PH: Microbiology of airway disease in patients with cystic fibrosis. Clin Microbiol Rev 1991; 4: 35-51.

3. Dimech WJ, Hellyar AG, Kotiw M, Marcon D, Ellis S, Carson M: Typing of strains from a single-source outbreak of Pseudomonas pickettii. J Clin Microbiol 1993; 31: 3001-3006.

4. Fujita S, Yoshida T, Matsubara F: Pseudomonas pickettii bacteremia. J Clin Microbiol 1981; 13: 781-782.

5. Fass RJ, Barnishan J: Acute meningitis due to a Pseudomonaslike group Va-1 bacillus. Ann Intern Med 1976; 84: 51-52.

6. Graber CD, Jervey LP, Ostrander WE, Salley LH, Weaver RE: Endocarditis due to a lanthanic, unclassified gram-negative bacterium (group IVd). Am J Clin Pathol 1968; 49: 220-223.

7. Wertheim WA, Markovitz DM: Osteomyelitis and intervertebral discitis caused by Pseudomonas pickettii. J Clin Microbiol 1992; 30: $2506-2508$.

8. Maroye P, Doermann HP, Rogues AM, Gachie JP, Megraud F: Investigation of an outbreak of Ralstonia pickettii in a pediatric hospital by RAPD. J Hosp Infect 2000; 44: 267-272.
9. Hsueh PR, Teng LJ, Pan HJ, Chen YC, Sun CC, Ho SW, Luh KT: Outbreak of Pseudomonas fluorescens among oncology patients. $J$ Clin Microbiol 1998; 36: 2914-2917.

10. CDC. Nosocomial Ralstonia pickettii colonization associated with intrinsically contaminated saline solution - Los Angeles, California, 1998. MMWR Morb Mortal Wkly Rep 1998; 47: 285-286.

11. Labarca JA, Trick WE, Peterson CL, Carson LA, Holt SC, Arduino MJ, Meylan M, Mascola L, Jarvis WR: A multistate nosocomial outbreak of Ralstonia pickettii colonization associated with an intrinsically contaminated respiratory care solution. Clin Infect Dis 1999; 29: 1281-1286.

12. Hancock REW: Resistance mechanisms in Pseudomonas aeruginos $a$ and other nonfermentative gram-negative bacteria. Clin Infect Dis 1998; 27 (Suppl. 1): 93-99.

13. Nordmann P, Poirel L, Kubina M, Casetta A, Naas T: Biochemicalgenetic characterization and distribution of OXA-22, a chromosomal and inducible class D ß-lactamase from Ralstonia (Pseudomonas) pickettii. Antimicrob Agents Chemother 2000; 44: 2201-2204. 